\title{
233 In the upper air passages
}

A The length of cervical trachea can alter in any one individual.

B The trachea is nearest to the skin at the 4th tracheal ring.

C The larynx descends during postnatal growth.

D The pleural dome is at risk during tracheostomy.

E The cricoid cartilage may be more easily palpated than the thyroid cartilage.

\section{In performing a tracheostomy}

A The head should be extended to a maximum degree.

B A vertical tracheal incision should be avoided in children.

C The tracheal incision should always include the first tracheal ring.

D The tracheostomy tube should fit tightly without a cuff.

E Tapes should be tied with the head in the neutral position.

\section{In the postoperative care of tracheostomy}

A A postoperative lateral cervical X-ray should be performed.

B Accidental decannulation after 2 weeks should be treated as an emergency.

C Copious mucus production indicates a pulmonary infection.

D A poorly positioned tracheostomy tube may produce a fatal haemorrhage.

E Mucus and crusts are the commonest causes of obstruction. 